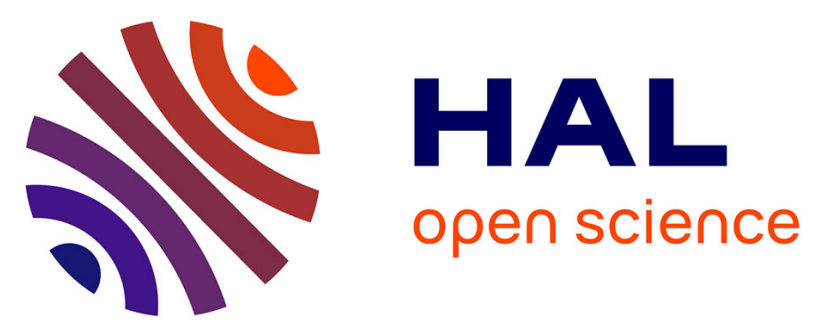

\title{
Numerical simulation of a non linear coupled fluid-structure problem by explicit finite element-finite volume coupling
}

\author{
Jean-François Sigrist, Christian Lainé, Bernard Peseux
}

\section{- To cite this version:}

Jean-François Sigrist, Christian Lainé, Bernard Peseux. Numerical simulation of a non linear coupled fluid-structure problem by explicit finite element-finite volume coupling. 2005 ASME Pressure Vessel and Piping Conference, Jul 2005, Denver, United States. 10.1115/PVP2005-71227 . hal-01008557

\section{HAL Id: hal-01008557 \\ https://hal.science/hal-01008557}

Submitted on 6 Nov 2018

HAL is a multi-disciplinary open access archive for the deposit and dissemination of scientific research documents, whether they are published or not. The documents may come from teaching and research institutions in France or abroad, or from public or private research centers.
L'archive ouverte pluridisciplinaire HAL, est destinée au dépôt et à la diffusion de documents scientifiques de niveau recherche, publiés ou non, émanant des établissements d'enseignement et de recherche français ou étrangers, des laboratoires publics ou privés.

\section{(c)(1)}

Distributed under a Creative Commons Attribution| 4.0 International License 


\title{
NUMERICAL SIMULATION OF A NON LINEAR COUPLED FLUID-STRUCTURE PROBLEM BY EXPLICIT FINITE ELEMENT-FINITE VOLUME COUPLING
}

\author{
Jean François SIGRIST \\ Service Scientifique et Technique \\ DCN Propulsion \\ 44620 LA MONTAGNE, France \\ jean-francois.sigristedcn. fr
}

\author{
Christian LAINE \\ Service Scientifique et Technique \\ DCN Propulsion \\ 44620 LA MONTAGNE, France
}

\author{
Bernard PESEUX \\ GéM - Pole Dynamique et Couplages \\ Ecole Centrale de Nantes - 1, rue de La Noë \\ 44321 Nantes Cedex 3, France
}

\begin{abstract}
The present paper deals with the numerical simulation of a coupled non linear tluid-stucture problem by explicit coupling between a finite element structure code and a finite volume fluid code. This numerical study is carried out in order to develop robust and general coupling with FE and CFD commercial code for indusirial applications.

A geometrically simple non linear coupled problem is presented in order to validate the numerical approach. The structure non linear problem is solved with a finite element technique, using a iteıative implicit algorithm for time integiation. The fluid problem is solved using standard numerical technigues (finite volume approach, implicit splitting operator scheme). The whole coupled problem is solved with a commercial CFD code: a dedicated FE structure code is developed in the CFD code together with coupling (in time, in space) procedures.
\end{abstract}

The proposed method is validated in the case of a incompressible inviscid tluid, for which the coupled problem is solved with an analytical solution. The present study gives a reference test case for a full scale fluid-sluucture model. Industrial appfications can now be considered by coupling commercial $\mathrm{FE}$ and FV codes with general coupling code.

\section{INTRODUCTION}

The present paper deals with the numerical simulation of coupled fluid-structure problem, in which the coupling process is bases on mechanical exchanges between each sub-problem. In the present study other fluid-struclure coupling phenomena, such as thermal effect, are not taken into account. At the fluid structure interface, lluid and structure arc coupled via lluid forces and structure displacement, as sketched by Fig. 1.

Over the past years many numerical methuds have been developed in order to solve linear and non linear coupled fluid- structure problems, using linite element and boundary element techniques [4][15] [16].

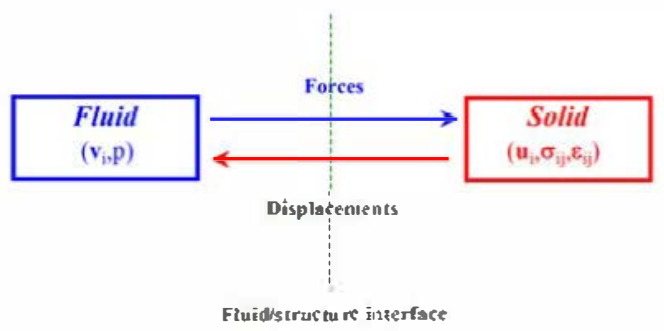

Fîgure 1. Mechanical Fluid/structure coupling [20]

Nowadays, the numerical approach tends to propose general coupling algorithms with specific solvers for each sub-problems, with various coupling strategies [5] [8] [17] [20], depending on the physical coupling phenomenon.

As the numerical design naval propulsion structures needs laking lluid/stucture inlcraclion inlo account, DCN Propulsion launched, with Ecole Centrale Nantes GéM, a R\&D study in order to apply numerical methods on industrial projects. From the academic point of view, the coupling process seems not to pose any paricular difficulties. From the industrial point of view, an investigation of the various aspects of the numerical fluid-structure coupling on a simple case is necessaly before considering a full-scale coupling with existing commercial codes for industrial structure design.

The fluid-structure coupled problem studied in the present paper is described by Fig. $2^{\prime}$. The numerical resolution of the coupled

\footnotetext{
${ }^{I} I t$ is an extension in the $3 D$ case of the non linear $2 D$ problem studied in a previous publication [2I].
} 
problem is performed using an explicit coupling techniques with a CFD fluid code and FE structure code. as represented by Fig. 3.

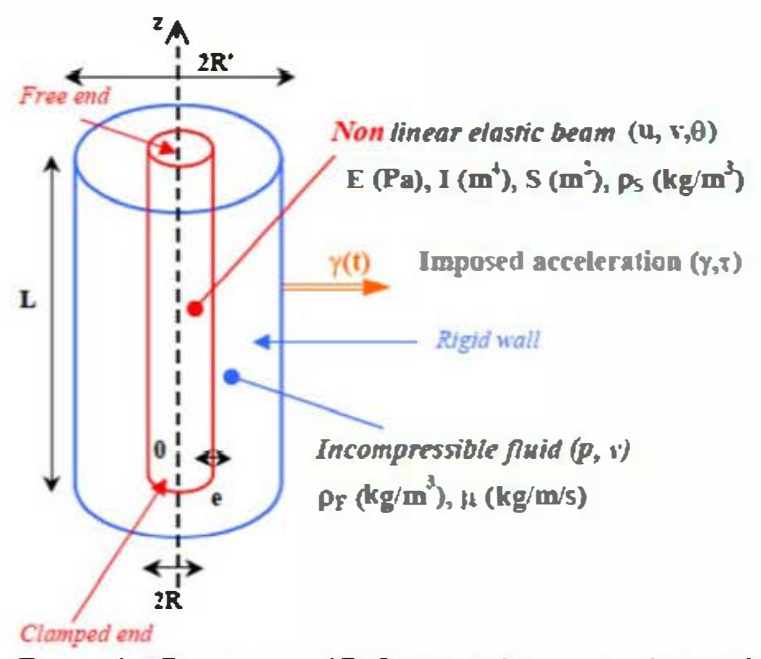

Figure 2. 1D structure-3D fluid non linear coupled problem

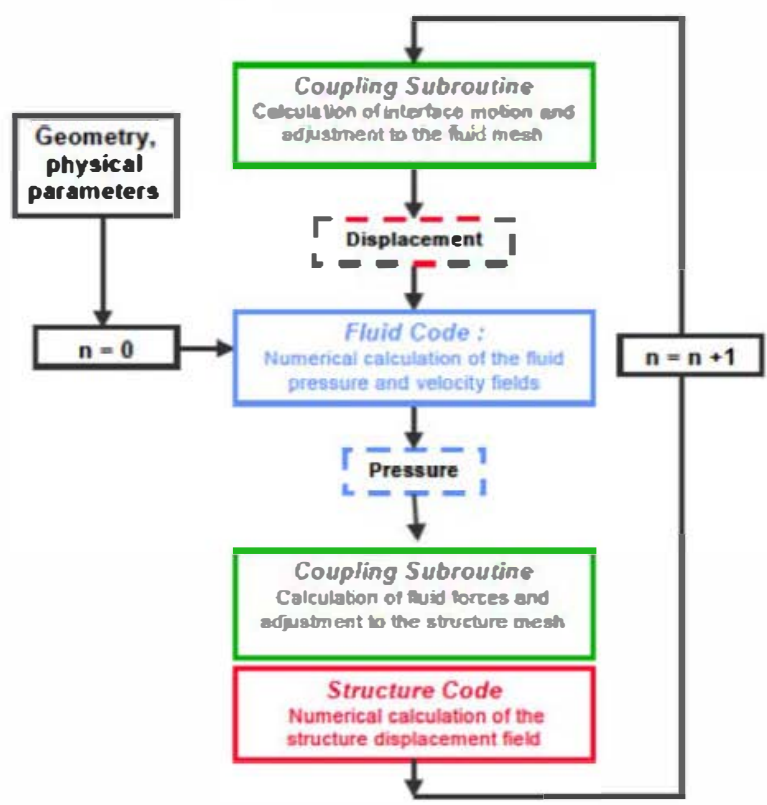

Figure 3. Fluid and structure code coupling: general principle

\section{STRUCTURE PROBLEM}

The structure problem is described by the following beam non linear equation of motion. taking into account geometrical non linearity [12] [26]. The coupled raction bending equation of motion are formulated in the relative frame as:

$$
\rho_{s} S \frac{\partial^{2} u}{\partial t^{2}}+\frac{\partial}{\partial z}\left\{E S\left[\frac{\partial u}{\partial z}+\frac{1}{2}\left(\frac{\partial v}{\partial z}\right)^{2}\right]\right\}=0
$$

$$
\rho_{s} S \frac{\partial^{2} v}{\partial t^{2}}+\frac{\partial^{2}}{\partial z^{2}}\left(E I \frac{\partial^{2} v}{\partial z^{2}}\right)-\frac{\partial}{\partial z}\left\{E S\left[\frac{\partial u}{\partial z}+\frac{1}{2}\left(\frac{\partial v}{\partial z}\right)^{2}\right] \frac{\partial v}{\partial z}\right\}=-\rho_{s} S \gamma
$$

The boundary conditions are:

$$
u_{z=0}=0 \quad v_{z=0}=0 \quad \frac{\partial v}{\partial z=0}=0
$$

for the beam clamped end. and:

$$
\left(\frac{\partial u}{\partial z}+\frac{1}{2}\left(\frac{\partial v}{\partial z}\right)^{2}\right)_{z=}=0 \quad \frac{\partial^{2} v}{\partial z^{2}=L}=0 \quad \frac{\partial^{3} v}{\partial z^{3}=L}=0
$$

for the beam free end.

\subsection{Space discretisatlon: fnite element method}

The numerical resolution of the structure problem is performed using the finite element method [1]. The variationnal foruulation of the non linear problem is obtained by multiplying Eq. (1) and (2) by virtual displacements $\delta u$ and $\delta$, integrating the equations over $[0 . L]$, using pant integration. leading to:

$$
\begin{aligned}
& \int_{0}^{L} \rho_{5} S \frac{\partial^{2} u}{\partial t^{2}} \delta u+\int_{0}^{L} E S \frac{\partial u}{\partial z} \frac{\partial \delta u}{\partial z}+\int_{0}^{2} \frac{E S}{2}\left(\frac{\partial_{v}}{\partial z}\right)^{2} \frac{\partial \delta u}{\partial z}=0 \\
& \int_{0}^{2} \rho_{s} S \frac{\partial^{2} v}{\partial t^{2}} \delta v+\int_{0}^{L} E I \frac{\hat{c}^{2} v}{\partial z^{2}} \frac{\partial^{2} \delta}{\partial z^{2}}+ \\
& \int_{0}^{2} E S\left[\frac{\partial v}{\partial z}+\frac{1}{2}\left(\frac{\partial v^{\prime}}{\partial z}\right)^{2}\right] \frac{\partial v \cdot}{\partial z} \frac{\partial \delta v}{\partial z}=-\int_{0}^{L} \rho_{s} S \gamma \delta v \cdot
\end{aligned}
$$

The space discretisation uses 2-nodes/3-degress of freedom beam finite element, as represented by Fig. 4. The unknown displacement are approximated with linear shape functions for " and cubic shape functions for $r$, that is witing $u=\left\langle N_{u}\right\rangle\{C\}$ and $v=\left\{N_{v}\right\}\{v\}$ with:

$$
\begin{gathered}
N_{\mathrm{u}}=\langle 1-s, s\rangle \\
N_{v}=\left\langle 1-3 s^{2}+2 s^{3}, l\left(s-2 s^{2}+s^{3}\right), 3 s^{2}-2 s^{3}, l\left(-s^{2}+s^{3}\right)\right\rangle(9)
\end{gathered}
$$

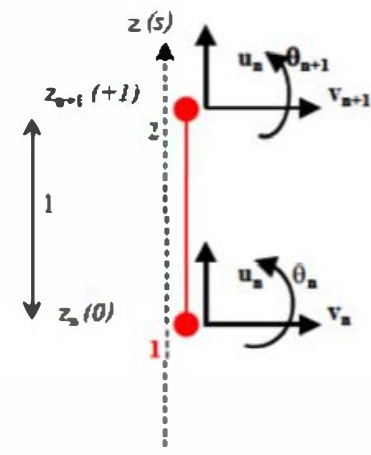

Figure 4. Beam finite element with two nodes, three degrees of freedom per node 
Using a GALERXIN method (i.e. miting an identical approximation for unlnown displacement $u, v$ and virtual displacement with the same shape finctions), the variationnal principle given by Eqs. (5) and (0) is written in the following discrete form:

$$
\left[\begin{array}{cc}
M_{u} & 0 \\
0 & M_{v}
\end{array}\right]\left\{\begin{array}{l}
\ddot{U} \\
\ddot{V}
\end{array}\right\}+\left[\begin{array}{cc}
K_{v} & 0 \\
0 & K_{v}
\end{array}\right]\left\{\begin{array}{l}
U \\
V
\end{array}\right\}+\left\{\begin{array}{l}
R_{u}(V) \\
R_{v}(U)
\end{array}\right\}=\left\{\begin{array}{c}
0 \\
\Phi_{v}
\end{array}\right\}
$$

In Eq. (10), the mass matrices are given by $\left.\left[M_{n}\right]=\int_{0}^{2} \rho_{s} s\left\{N_{-}\right\} N_{-}\right\rangle$. and $\left.\left[M_{v}\right]=\int_{0}^{t} \rho_{s} s\left\{N_{v}\right\} N_{v}\right\}$, the stiffness matrices are expressed as $\left[\boldsymbol{K}_{\mathbf{.}}\right]=\int_{0}^{I} E S\left\{\frac{\partial N_{u}}{\partial z}\right\}\left\langle\frac{\partial N_{u}}{\partial z}\right\rangle$ and $\left[\boldsymbol{K}_{v}\right]=\int_{0}^{L} E I\left\{\frac{\partial^{2} N_{v}}{\partial z}\right\}\left\langle\frac{\partial^{2} N_{v}}{\partial z}\right\rangle$, and the loading vector is turitico $\left\{\Phi_{0}\right\}=-\int_{0}^{2} \rho_{s} S \gamma\left\{N_{v}\right\}$. With the shape functions defined by Eqs. (8) and (9). these matrices can be analytically calculated. The non linear terms are:

$$
\begin{array}{r}
\left\{R_{\cdot}(V)\right\}=\int_{0}^{L} \frac{E S}{2}\left\{\frac{\partial N_{u}}{\partial z}\right\}\langle V\rangle\left\{\frac{\partial N_{v}}{\partial z}\right\}\left(\frac{\partial N_{v}}{\partial z}\right)\{V\} \\
\left\{R_{v}(U, V)\right\}=\int_{0}^{2} E S\left\{\frac{\partial N_{v}}{\partial z}\right\}\left\langle\left(\frac{\partial N_{u}}{\partial z}\right)\{U\}+\right. \\
\left.\frac{1}{2}\langle V\rangle\left\{\frac{\partial N_{v}}{\partial z}\right\}\left(\frac{\partial N_{v}}{\partial z}\right)\{V\}\right]\left(\frac{\partial N_{v}}{\partial z}\right)\{V\}
\end{array}
$$

\subsection{Time integration: non linear implicit scheme}

The dynanic non linear system given by Eq. (10) can be formulated as follows:

$$
M \ddot{X}+K(X)=\Phi-M
$$

with $K(X)$ denoving the non linear stiffness. D external forces'. and $-M T$ being the inertial term ${ }^{3}$. If fluid forces are taken into account. ( $)$ is obtained from the discrete form of the following virtual work $\delta W=\int_{\Gamma(s)} \delta \bar{U} \cdot \overline{\bar{\sigma}} \cdot \vec{n} d \Gamma$ where $\overline{\bar{\sigma}}$ is the fluid stress tensor. $\bar{n}$ is the outer nomul at the fluid-structure interface $\Gamma$.

The numerical time integration of Eq. (12) can be performed with classical techniques. using explicit or implicit techniques.

\footnotetext{
${ }^{2}$ In the present paper, the enenal forces are the fluid forces, which will be compused from a CFD cade using a finite volume approach, see \$. 2 .

${ }^{3}$ The proposed -MT notation in Eq. (12) is an alsernative woy of writing the dyamic loading $\left\{\Phi_{v}\right\}$ appearing in Eg. (10).
}

In explicit techniques [7]. a three points numerical scherne is used to eraluate the acceleration at time $t_{n}$. For instance, the centered finite differcnce scheme gives the approximation:

$$
\ddot{X}_{n}=\frac{X_{n+1}-2 X_{n}+X_{n+1}}{\delta t^{2}}
$$

Using the expression of Eq. (14) in the general equation of motion leads to:

$$
X_{n+1}=\delta t^{2} M^{1} \times\left[\phi_{n}-M \Gamma_{n}-K_{n}\right]+2 X_{n}-X_{n 1}
$$

In Eq. (15), all terms in the right side are known. allowing a straightforward calculation of $X_{n+1}$. The explicit technique can be coupled with the finite volume approach of the fluid problem in a staggered resolution of the coupled problem. Though the implementation of the coupling is rather easygoing in the explicit approach. it suffers from stability limitations. This leads to the use of an implicit technique.

In implicit technique [23]. a two points numerical scheme is used; the NENMLARK scheme gives for example:

$$
\ddot{X}_{\infty+1}=\frac{X_{n+1}-X_{n}}{\beta \delta t^{2}}-\frac{\dot{X}_{n}}{\beta \delta t}-\left(\frac{1}{2 \beta}-1\right) \ddot{X}_{n}
$$

The substitution of Eq. (16) in the equation of motion leads to an implicit equation in terms of the unlnown displacement $X_{n+1}$. An iterative procedure based on fixed point algorithm is performed to obtain $X_{n+1}$. The algorithm starts with a linear calculation (with the lineas part of $K(X)$ ), which gives an estimation of the structure displacement $X_{a+1}{ }^{\circ}$. Iterations are performed with the following approximation:

$$
K\left(X_{a+1}^{i+1}\right)=K\left(X_{a+1}^{i}\right)+\left(\frac{\partial K}{\partial X}_{n+1, i}\right) \cdot\left(X_{a+1}^{j+1}-X_{n+1}^{\prime}\right)+O\left(\Delta X_{a+1}^{j+1^{2}}\right)
$$

which gives:

$$
\begin{aligned}
X_{n+1}^{i+1}= & \bar{K}_{n+1, i}{ }^{1} \times\left[\Phi_{n+3}+K\left(X_{n+3}^{\prime}\right)+\left(\frac{\partial K}{\partial X} X_{a+1 ;}\right) \cdot X^{\prime}+\right. \\
& \frac{M}{\beta \delta t^{2}} X_{n}+\frac{M}{\beta \delta t} \dot{X}_{n}+M\left(\frac{1}{2 \beta-1}\right) \ddot{X}
\end{aligned}
$$

where $\tilde{K}_{a+L i}=\frac{M}{\beta \delta t^{2}}+\left(\frac{\partial K}{\partial X}_{a+L i}\right)$. The iteration process is stopped when a convergence criterion is satisfied: a simple criterion can be written as:

$$
\frac{\left\|X_{n+1}^{i+1}-X_{++1}^{i}\right\|}{\left\|X_{m+1}^{i}\right\|} \leq \varepsilon
$$


where $\varepsilon \ll 1$. This approach is equivalent to a NEWTON procedure, and converges under known assumptions. The non linear scheme requires the calculation of the tangent stiffoses matrix $\frac{\partial K}{\partial X}$. In the present case, the matrix is expressed as:

$$
\frac{\partial K}{\partial X}=\left[\begin{array}{cc}
K_{v} & \frac{\partial R_{z}}{\partial V} \\
\frac{\partial R_{v}}{\partial U} & K_{v}+\frac{\partial R_{v}}{\partial V}
\end{array}\right]
$$

with:

$$
\begin{aligned}
& \frac{\partial R_{0}}{\partial V}=\int_{0}^{z} E S\left\{\frac{\partial N_{u}}{\partial z}\right\}\langle V\rangle\left\{\frac{\partial N_{v}}{\partial z}\right\}\left\langle\frac{\partial N_{v}}{\partial z}\right\rangle \\
& \left.\frac{\partial R_{v}}{\partial U}=\int_{0}^{2} E S\left\{\frac{\partial N_{v}}{\partial z}\right\}\left(\frac{\partial N_{v}}{\partial z}\right)\left\{\frac{\partial N_{v}}{\partial z}\right\} V\right)
\end{aligned}
$$

and:

$$
\begin{aligned}
& \frac{\partial R_{v}}{\partial V}=\int_{0}^{L} E S\left\{\frac{\partial N_{v}}{\partial z}\right\}\langle V\rangle\left\{\frac{\partial N_{v}}{\partial z}\right\}\left\langle\frac{\partial N_{v}}{\partial z}\right)\left\langle\frac{\partial N_{v}}{\partial z}\right)\{V\}+ \\
& \int_{0}^{L} E S\left\{\frac{\partial N_{v}}{\partial z}\right\}\left(\frac{\partial N_{v}}{\partial z}\right)\{U\}\left\langle\left(\frac{\partial N_{v}}{\partial z}\right)\right. \\
& +\int_{0}^{L} \frac{E S}{2}\left\{\frac{\partial N_{v}}{\partial z}\right\}\langle V\rangle\left\{\frac{\partial N_{v}}{\partial z}\right\}\left(\frac{\partial N_{v}}{\partial z}\right)\{V\},\left(\frac{\partial N_{v}}{\partial z}\right)
\end{aligned}
$$

The non-linear terms of these matrices are computed using a GAUSS-LEGENDRE procedure [1], that is using a numerical integration scheme based on the following approximation:

$$
\int_{1}^{+1} \psi(\zeta) d \zeta \approx \sum_{l=1}^{1} w_{l} \psi\left(\zeta_{l}\right)
$$

with $\zeta_{l}$ Gauss integration point and $w$ the associated integration weight. The numerical scheme (24) using $L$ points is exact for the polynomial finctions of degree $2 L-1$ : the numerical integration procedure used in the present case mill be based on a 5 points scheme. Furthermore, the iterative scheme given by Eq. (18) needs inverting matrix $\frac{M}{\beta \delta t^{2}}+\left(\frac{\partial K}{\partial X_{*+1, j}}\right)$ at each time step. which is done with a LU factorization.

The above finite element procedure is implemented as FORTRAN subroutines, which will be integrated as a simple structural code in the commercial CFD code used for the numerical resolution of the fluid problem. Coupling procedures preseated in $\S .3$ will also be implemented as FORIRAN subroutine of the CFD code.

\section{FLUID PROBLEM}

The fluid problem is described by the general NAVIFR-STOKxs equation. The conservation equation are integated over a moving control volume $\Omega(r)$ of boundary $\hat{\sigma} \Omega(r)$. using the LIEBNITZ rule and the GAUSS theorem. This leads to the global conscriation equation over an arbitrary control volume [25].in ALE formulation [19]:

- Mass conservation

$$
\frac{d}{d t}\left(\int_{\Omega(t)} \rho d \Omega\right)+\int_{n(t)} \rho\left(v_{j}-v_{j}^{*}\right) d n_{j}=\int_{\Omega(r)} S_{\rho} d \Omega
$$

- Momennm conservation

$$
\begin{aligned}
& \frac{d}{d r}\left(\int_{\Omega(s)} \rho r_{j} d \Omega\right)+\int_{(\Omega)(s)} \rho r_{j}\left(v_{j}-v_{j}\right) d n_{j}= \\
& -\int_{\Omega(n)} p d n_{j}+\int_{\Omega(n)} \mu\left(\frac{\partial r_{i}}{\partial x_{j}}+\frac{\partial v_{j}}{\partial x_{j}}\right) d n_{j}+\int_{\Omega(n)} S_{n} d \Omega
\end{aligned}
$$

In Eqs. (26) and (26). $S_{\rho}$ and $S_{v_{j}}$ stand respectively for mass and momentum sources: the fluid problem can then be formulated in the moving frame. The fluid unknown are the pressure and velocity fields $p, v_{j}$, but as the conservation equation are integrated over a moving control volume. Eqs. (25) and (26) show another unknown fluid that is the grid velocity $v_{i}^{*}$ : another equation bas to be used to close the fluid problem. This supplementary equation is the space law conservation [6] which is written:

$$
\frac{d}{d t}\left(\int_{\Omega(t)} d \Omega\right)=\int_{\omega(t)} v_{j}^{*} d n_{j}
$$

The fluid problem is then fully characterized by Eqs. (25) to $(27)^{4}$. Additional boundary conditions are to be taken into account such as imposed pressure or velocity. The coupling with the structure problem is expressed as $\left.\bar{v}\right|_{r}=\left.\frac{\partial \vec{u}}{\partial t}\right|_{\tau}$ where $\bar{u}$ is the sinicture displacement and $\Gamma$ is the fluid-structure interface.

\subsection{Space discretisation: finite volume method}

The general form of an integrated conservation equation for a fluid unknown $\phi$ is the following one:

$$
\frac{d}{d t}\left(\int_{\Omega(t)} \rho \phi d \Omega\right)+\int_{\alpha \Omega(t)} \rho\left(v_{j}-v_{j}^{*}\right) \phi d n_{j}=\int_{\Omega(t)} S_{\phi} d \Omega+\int_{\alpha(t)} \Gamma \frac{\partial \phi}{\partial x_{j}} d n_{j}
$$

Equation (28) is discretised using a finite volume method. The fluid domain is divided in elementary fluid cells (Fig. 5 gives a typical representation of a computational grid for a $2 D$ cartesian problem). for which a integrated conservation equation is written. A moving mesh teclumique is used. the elenwentary grid velecity is deduced from known node displacement in order to satisfy Eq. (27)

\footnotetext{
II the case of a compressible fluid, the fiuid state lars $\rho(p)$ allows a closure of the problem.
} 
[6]. The grid velocity $v_{i}^{*}$ appearing in the right side of Eq. (28) becomes then a source tem on the left hand side. Integrated conservation equation are written for each cell: $P$ is the cell center. $W W . W . S W . S, S E, E, E E . N E, N$ are the centers of neighboring cells.

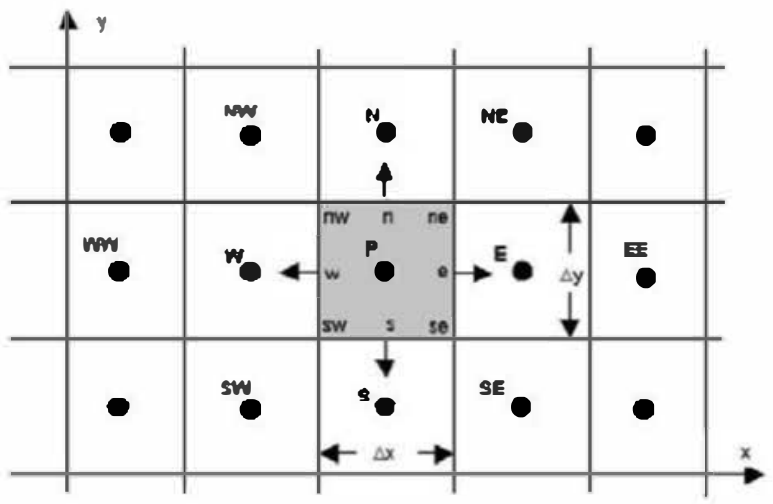

Figure 5. 2D fluid computational grid

The time dependant in Eq. (28) are approximated by an EULER scheme. i.e. writing:

$$
\frac{d}{d t}\left(\int_{\Omega,(t)} \rho \phi d \Omega\right) \approx \frac{\rho \Omega}{\delta t}\left(\phi_{P}^{n+1}-\phi_{P}^{n+1}\right)
$$

The convective and diffusive terms are calculated using finite difference approximation, leading to the global expression [9]:

$$
\int_{\mathcal{M}_{p}(t)} \rho v_{j} \phi-\Gamma \frac{\partial \phi}{\partial x_{j}} d n_{j} \approx \sum_{M} C_{M}^{n+1} \phi_{M}^{n+1}-D_{M}^{n+1} \phi_{M}^{n+1}
$$

where $M$ stands for the neighboring points of cell $\Omega p$.

Taking into account Eqs. (29) and (30) leads to the following algebraic non linear system $[A(\phi)] \phi\}=\{b\}$, that is:

$$
A_{P} \phi_{P}^{n+2}=\sum_{M} A_{P} \phi_{P}^{n+1}+b_{P} \phi_{P}^{n}
$$

\subsection{Time integration: PISO algorithm}

From discretisation practices presented above, it is possible to produce the following algebraic system resulting from the finite volume discretisation of the momentum equation. Eq. (32). and Posso. equations. Eq. (33).

$$
\begin{aligned}
& A_{p} v_{, p}^{\infty-1}=H\left(v_{i}^{n-1}\right)+D\left(p^{n-1}\right)+S_{v_{i}}^{n-1} \\
& A_{P} p_{P}^{n+1}=G\left(p^{n+1}\right)+S_{p}\left(v_{i}^{n+1}\right)
\end{aligned}
$$

Equations (32) and (33) couple the pressure and velocity unknowns. An implicit iterative scheme is used to calculate the new values of the velocity and pressure field [13]. The algorithm statts with an estimation of the velocity field. by solving the following non linear system. obtained from Eq. (33) by using the pressure field computed at the previous time step:

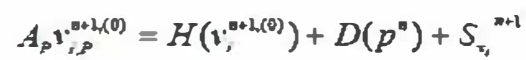

Equations (32) and (33) are then used to produce successive correction. by using the iterative relations:

$$
\left\{\begin{array}{c}
A_{P} v_{i, p}^{n+1,(q+1)}=H\left(v_{i}^{n+1,(q)}\right)+D\left(p^{n+1,(q)}\right)+S_{v_{i}}{ }^{n+1} \\
A_{p} p_{P}^{n+1,(q)}=G\left(p^{n+1,(q)}\right)+S_{p}\left(v_{i}^{n+1,(q)}\right)
\end{array}\right.
$$

The iterative scheme is stopped when a convergence criterion is satisfied or when a maximum number of inner iterations is reached.

The fluid problem is solved with the numerical principles expose before. using the commercial CFD code Star-CD [28]. An elementary validation of the moving mesh technique and fluid force calculation is performed in the elementary case of an oscillating cylinder in a confined annular space. The FRrIz model [10] gives the added mass coefficient for small amplitude motions in the case of a perfect fluid. Figure 6 gives a comparison of the analytical and computed added mass coeficient for several confinement ratios ${ }^{5}$.

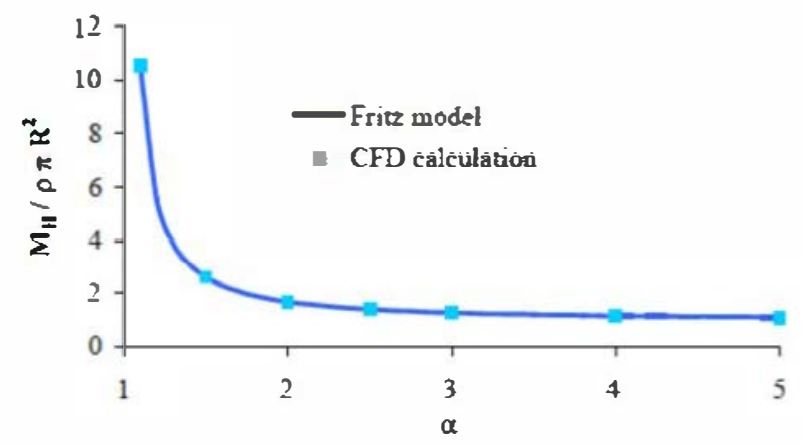

Figure 6. Elementary validation of the moving mesh and pressure force calculation with the Star-CD CFD code

\section{COUPLED PROBLEM}

The fluid and structure problem are solved with the numerical principles presented in the previous paragraphs. The numerical methods for fluid are available as such in the CFD code STAR-CD. which will be used for the present study: the structural code will be implemented in the STAR-CD code as FOTRAN subroutines, together with coupling pracedures.

The numerical solving strategy of the STAR-CD code does not allow numerical exchanges within a time iteration with PISO

\footnotetext{
SA numerical stud: currently in progress focuses on the calculation of added mass and added damping cosfjcient in the case of a viscous fiuid. Phaliminan' resuls show a good agreement with the anahrical model from CHEV and comparison with other numerical resulis [3] will be carried our.
} 
algorithm: coupling with a structure code will thus be possible only with an explicit technique as detailed bellow.

\subsection{Coupling in time}

As mentioned before, the time coupling strategy will be based on a swg gered. explicit coupling procedure, which is represented by Fig. 7.

At time step $i_{n}$, stucture displacement $u_{n}$ is known. From previous fluid geometry $\Omega_{n}$. it is possible to calculate the new fluid geomety $\Omega_{n}$ and solve the space law conservation equation to obtain the grid velocity field $v_{n}$ (step [1] in the coupling procedure). The calculations proceed uith the numerical resolution of the fluid problem on fluid domain $\Omega_{n}$. requiring inner iterations for the PISO algorithm (step [2]). Fluid forces on the fluid/stracture $\varphi_{n}$ interface are then decuced and transferred on the structure problem (step [3]). The advance in time for the stuuctural problem is then carried out, with inner iteraions for the implicit scheme (step [4]).

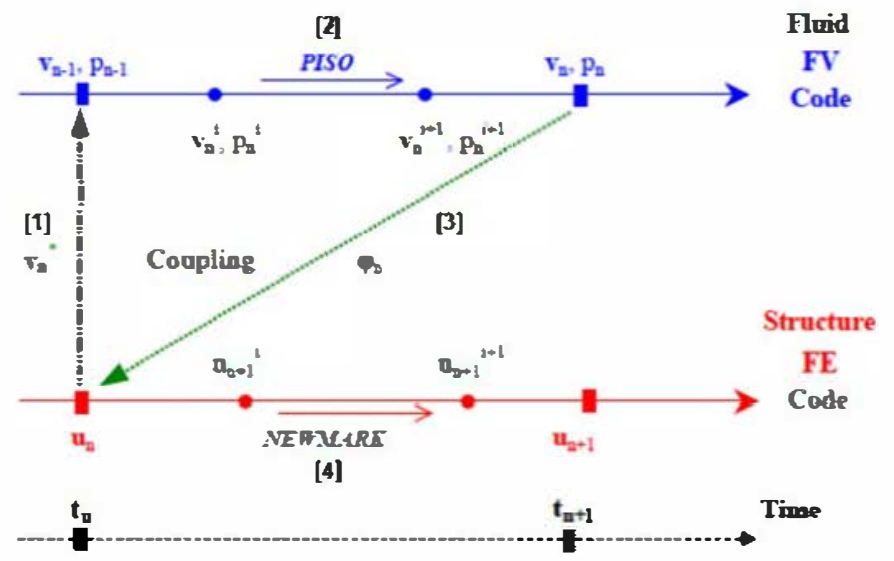

\section{Figure 7. FE structure code and FV fluid code explicit time coupling}

From the structure point of view, the implicit approach requires the knowledge of external forces at time step $t_{n+1}$. whereas the explicit coupling procedure gives fluid forces from $r_{n}$, to $r_{n}$; a linear extrapolation is used to a better predicion of the fluid forces: the external forces used in the surcture advance are $\tilde{\varphi}_{n}=2 \varphi_{n}-\varphi_{n} 1$.

For small time step size. or small mesh displacement, the computed fluid forces show high frequency numerical oscillations. This can induce numerical instabilities for the structure part. This problem is addressed by introducing a filtering process within the coupled procedure ${ }^{6}$. A first order filter is used. It is described by its ronsfer funcrion:

\footnotetext{
6 From a numerical point of view, she existence of numerical ascillotions in the com pured fiuid forces can also induce numerical ssuchore instabilities, on in implicit coupling rechniques. Mam authors use in this cose a blending factor approach. The compused fivid forces are corrected with previous
}

$$
H(p)=\frac{1}{\tau \cdot p+1}
$$

where $p$ is the LAPIACE operator, and $\omega_{0}=2 \pi / \tau$ is the filtering pulsation. Using a bilinear transform of the LAPLACE variable $p$, that is $p=\frac{2}{\delta} \cdot \frac{z-1}{z+1}$, and using the z-transform [24]. one deduces the following discrete relation:

$$
\dot{\varphi_{n}}=\frac{\delta t}{2 \tau+\delta t} \cdot\left(\varphi_{n}+\varphi_{n 1}-\dot{\varphi}_{n 1}\right)+\frac{2 \tau}{2 \tau+\delta t} \cdot \dot{\varphi}_{n 1}
$$

where $\varphi$ and $\varphi^{*}$ are the non-filtered and filtered discrete values, $\delta t$ is the tinue step size ${ }^{7}$.

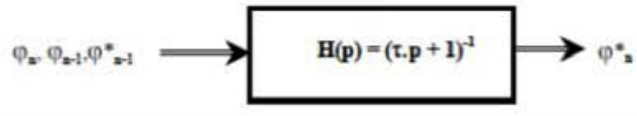

Figure 8. Discrete numeric filter

\subsection{Coupliag in space}

The space coupling procedure handles numerical exchanges between the fluid and structure problems. Structure displacement are transferred from the structure mesh to the fluid mesh. and fluid forces are projected from fluid boundaries to structure mesh. as sketched by Fig. 9.

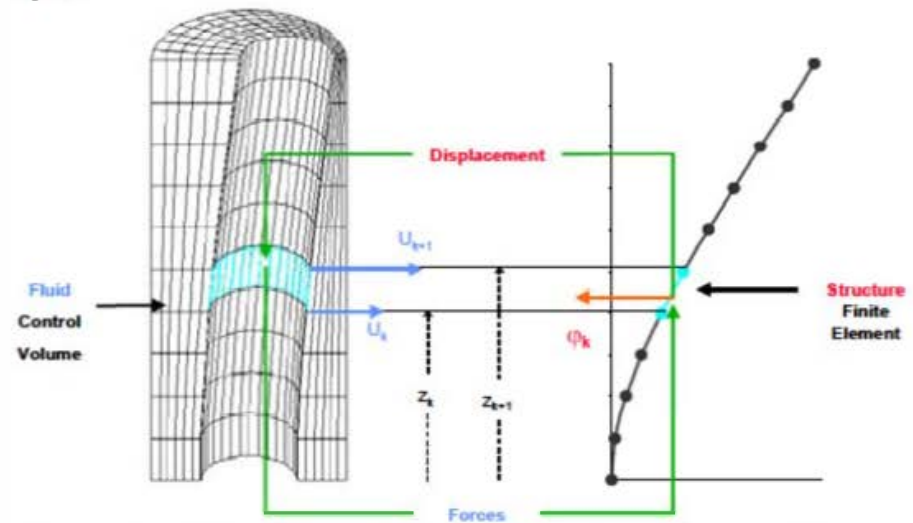

Figure 9. FE structure code and F fluid code space coupling

As fluid and sructure meshes can be very different (a few finite clement are enough to describe the structure dynamic, an important

forces, for inssance as $\hat{\varphi}_{n} \quad 6 p_{n}+(1 \quad \theta) \varphi$. wish $0<\theta<1$ [I]. This conection is aguivalent to the filcering/extropolation procedure used in the presentshid:

In she $2 D$ case, shis numerical meament proved to be afficient and pracise. even when fuid forces ore dominant over structure imer stiffiness forces [21].The major drowback of this technique lies on its empirical approach sereral calcularion with various filsering fiequencies have so be penformed in order so demonstrate thas numerical results are not affected fip so a cortain point) bu this numerical approach. 
number of fluid finite volume are required to solve the fluid problem. with local refinement to describe boundary layer). the space coupling procedure uses interpolation techniques in the displacement force exchanges beru een the nvo sub-p!oblems [ $[\underline{2}]$

Since the fluid problem is re-meshed at each time step to take into account structure deformation. the fluid inite volume can undergo great shape deformation. Many re-meshing coupling techniques can be develop to preserve the fluid mesh quality [22]. As the fluid geomery is rather simple in the present case. it is possible to develop a re-meshing procedure that produces little deformation of the fluid cells. based on a purely geometrical approach of the problem.

Figure 10 shous a 2D half slice of the fluid mesh. in the initial state and a deformed state; fluid cells length ratios are preserved even for large amplitude inner cylinder motion ${ }^{8}$.
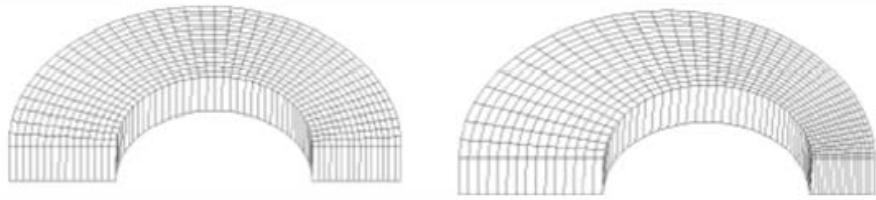

Initial mesh
Deformed mesh
Figure 10. Initial and deformed mesh

\section{VALIDATION OF THE COUPLING TECHNIQUE}

The numerical coupling procedure is validated in the case of a perfect incompressible fluid. Under these assumprions, a linearisarion of the NAVIER-STOKES equations leads to the following formulation of the fluid problem, in the non deforming relative frame [11]:

$$
\begin{gathered}
\Delta p=0 \\
\frac{\partial p}{\partial z_{(=0)}}=\frac{\partial p}{\left.\partial z_{(z)}\right)}=0 \\
\frac{\partial p}{\partial r_{(r)}}=-\rho_{\varepsilon} \gamma \cos \theta
\end{gathered}
$$

Coupling conditions with the smucture problem are wrinen as:

$$
\begin{aligned}
& \varphi_{k}(z)=-\int_{0}^{2 \pi} p(R \cdot \theta \cdot z) \cos \theta R d \theta \\
& \frac{\partial p}{\partial r_{(r z)}}=-\rho_{F}\left(\frac{\partial^{2} v}{\partial t^{2}}+\gamma\right) \cos \theta
\end{aligned}
$$

The fluid problem has an analytical solution that can be written in terms of pressure, from which fluid force on the elastic beam is decuaced an written as:

\footnotetext{
${ }^{8}$ For very large displacement, i.e. when the inner gylinder almost comes mo contaci with the outer one, the propased re-meshing rechnigue will fail lother anes too !...) so preserve mesh qualin: In this case a numerical rechnigue based on birth/deash fluid cells approach would be useful. Such a meshod is arvilable in the SIAR-CD code [28] and is currenthy investigated in the preans coupled problers.
}

$$
\begin{aligned}
& \varphi_{1}(z)=-\pi R \sum_{i=}^{5} P_{i}(R) \times \cos \left(q_{i}=\right) x \\
& \int_{0}^{1} \frac{\hat{c}^{2} v}{\partial r^{2}}(\zeta, l) \cos (\varphi, \zeta) d \zeta+\rho_{\xi} \pi R^{2} \gamma
\end{aligned}
$$

This leads to the calculation of an added mass matrix $M_{3}$ in the left hand side of structure equation of motion and a displaced fluid force vector $M_{\&} \Gamma$ on the right hand side:

$$
\left(M+M_{h}\right) \ddot{Y}+K(X)=-\left(M-M_{d}\right) \Gamma
$$

The structure problen taking into account fluid effect is then solved with numerical techniques presented in $\S .1$. The geomerrical and physical characteristics of the coupled problem are $R=0.1 \mathrm{~m}$. $R^{\prime}=0.2 \mathrm{~m} . I=1 \mathrm{~m}, e=0.02 \mathrm{~m} . \rho_{S}=8000 \mathrm{~kg} \mathrm{~m}^{3} . E=6.04 \times 10^{8}$ $\mathrm{Pa}$. The whole system is subjected to a sine wave acceleration:

$$
\gamma(t)=\left\{\begin{array}{ccc}
\gamma \times \sin \left(2 \pi \frac{t}{\tau}\right) & \text { if } & 0 \leq t \leq t \\
0 & \text { if } & t<t
\end{array}\right.
$$

Figure 11 shows the analytical solution and CFD calculation with explicit FEFV coupling in terms of bean free end displacement. The parameters of the imposed acceleration is are $\gamma=50 \mathrm{~m} / \mathrm{s}^{2} . \tau=100 \mathrm{~ms}$. The numerical results show a good agreement between the two methods, which validates the explicit coupling procedure.

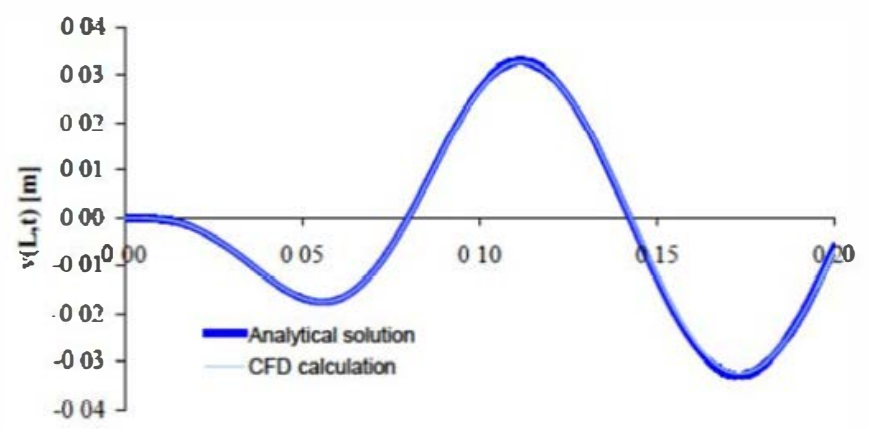

t ls]

Figure 11. Non linear beam response: free end displacement $v(L, t)$ computed mitb the analytical solution and CFD calculation with non linear $F E / F V$ coupling technique

Figure 12 gives a comparison of two coupled calculation with the previous acceleration parameters: the elastic beam is coupled with a light and heavy fluid. The numerical simularions illustrates the added mass and displaced mass effect: the period of oscillation is increased the amplitude oscillation decreased in the coupling with the heasy fluid. compared to the coupling with the light fluid. 


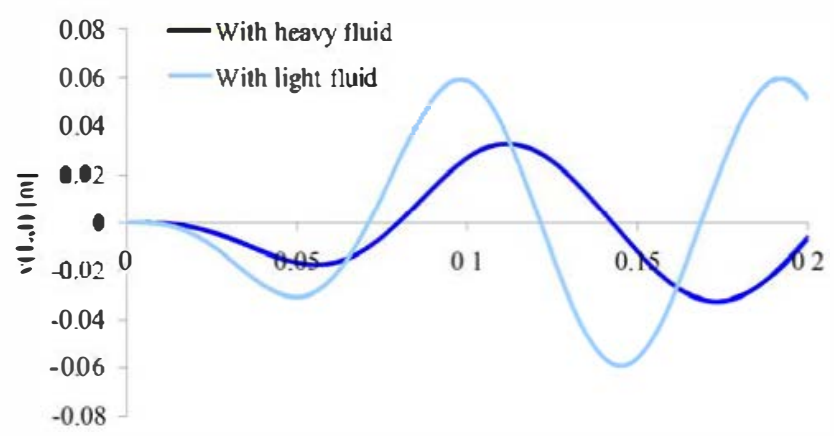

$t[s]$

Figure 12. Non linear beam response free end displacement $v(L, t)$ computed with CFD calculation. Elastic beam coupled with water $\left(\rho=1000 \mathrm{~kg} / \mathrm{m}^{3}\right)$ and with air $\left(p=1 \mathrm{~kg} / \mathrm{m}^{3}\right)$

\section{CONCLUSION}

A general explicit coupling between a structure finite element code and a fluid finite volume code has been presented and applied to the numerical simulation of a simple non !inear fluid-structure coupled problem.

Various aspect of the coupling procedure have been detailed: numerical methods for fluid and structure sub-problems. coupling practices, including explicit staggered time coupling, fluid remeshing strategy, data exchanges with numerical space interpolation and time filtering techniques.

The procedure is validated in the simple case of a perfect inviscid fluid with a numerical solution of the couplcd problem. The numerical fluid/structıre simulation with a more general fluid model can be considered. Current numerical studies lying on the exposed principles are performed for various cases (viscous fluid, with two fluid phases, elc...).

The present study gives a reference test case for a fulI scale fluid-structure model. Coupling comunercial FE code (such as ANSYS) and FV code (such as STAR-CD) with general coupling code (such as $\mathrm{MpCCl}$ ) lor industrial applications can now be considered.

The industrial application under consideration are related to coupled problems where the fluid-structure system, initially at rest, is excited by imposed displacement or acceleration. Fluid-structure dynamic are govemed by added mass and damping effect, lor which a numerical explicit resolution gives good results, without too a heavy modeling and computational cost. For coupled problems in which fluid flow induces continuous energy exchanges from fluid to structure lea ding to structure instabilities, explicit techniques might not be effective. Further studies will tend to investigate implicit coupling methods with appropriate numerical tools and numerical strategies and pertorm industrial calculation in such cases.

\section{REFERENCES}

[1] KJ. BATHE. Finite Element Precedures in Engineering Anahysis. PrenticeHall, Englewood Cliffs, 1982
[2] A. BECKERT. Coupling Fluid (CFD) and Structural (FE) models Using Finite Intespolation Elements. Aerospace Science and technology, 4 (1), 1322, 2000.

[3] 2. Bendedou, E. Longate, A. Amobes, M. Soull. Steam Generator Tube Vibrations Experimental determination versus ALE Computation of Fluidelastic Forces. Pressurc Vessel and Piping, Cleveland, 20-24 July 2003.

[4] F. Berot, B. Peseux. Vibro-A coustic Behavior of Submersed Cylindrical Shells: Analytical Formulation and Numerical model. Journal of Filuids \& Siructures, 12, 959-1 03. 1998

[5] F. Casadei, J.P. Halleux, A. Sai.a, F. Chille. Transiant Fluid Struclure Interaction Algorithm for I.arge Industrial Applications. Computer Methods in Applicd Mechanical Enginec ring. 190, 3081-3110, 2001.

[6] 1. Demirdzic, M. Peric. Space Conservation law in Finite Volume Calculations of Fluid Flow. International Journal for Numerical Methods in Fluids, 8, 1037-1050, 1988.

[7] M.A. DOKaINISH, K. Subbaraj. A Survey of Direct Time-Integration Methods in Computational Dynamics-I: Explicit Methods. Computer \& Structures, 32, 1371-1386, 1989.

[8] C. Farhat, M. Lesoinne, N. Maman. Mixed Explicit/mplicit Time Integration of Coupled Aeroelastic Problems. International Journal of Numerical Methods in Fluids, 21, 807-835, 1995.

[9] J.H. Ferziger, M. PerIC. Computational Methods for Fluid Dinamics. Springer-Verlag, 1999

[10] R.J. FrITz. The Effect of Liquids on the Dynamic Motion of Immersed Solids. Journal of Engineering for Industry, 167-173, 1972.

[11] R.J. GIBERT. Vibration des structures. Imeraction avec les fuides. Sources d'excitation aléatoires. Collection de la Direction des Eludes et Recherches d'Electricté de lirance, vol. 69, Eyrolles, 1986.

[12] 3. HINU. Y. YUSHIMURA, N. ANANTHANARAYANA. Vibration Analysis of Non-Linear Beams Subjected to a Moving Load Using the Finite Element Method. Journal of Sound and Vibration, 100, 477-491, 1985.

[13] R.I. IsS.4. Solution of The Implicitly Discretised Fluid Flow Equation by Operator Splitting. Journal of Compulational Phvsics, 62, 40-65, 1985

[14] E. DE LANGRE. Fluides et Solides. Les éditions de l'Ecolc Polytechnique, 2002.

[15] J. MAKERLE. Fluid-Structure Interaction Problems, Finite Element Approach and Boundary Élements Approaches. A Bibliography. Finite Elements in Analvsis and Design, 31, 231-240. 1999.

[16] Ii. J.P. Merand, R. OHAYON. Fluid Structure Interaction. Wiley \& Sons, 1995.

[17] I.M.T PeNrose, C.3. Staples. Implicit Fluid-Structure Coupling for Simulation of Cardiovascular Problems. International Journal for Numerical Methods in Fluids, 1, 1-13,2001.

[19] J. Sarrate, A. Huerta, J. Donea. Arbitrany Lagrangian Eulerian Formulation for Fluid Mulli-Rigid Bodies Inseraction Problems. Co mputation Mechanics, New Trend and Applications, 1998.

[20] M. SChäFtr. Coupled Fluid-Solid Prablems Survey on Numerical Approaches and Applications. Pressure Vessel and Piping. Cleveland, 20-24 July 2003

[21] J.F. Sigrist et al. Numerical Simulation of Fluid Sinucture Problems by Coupling Fluid Finite Volume and Structure Finite Element or Modul Approach. Flow Induced Vibrations. Paris, 5-9 July 2004.

[22] R.M.C. So, Y. Lu, Y.G. LAJ. Mesh Shape Prasenation for FlowInduced Vibrations Problems. International Mechanical Enginøering Congress and Exposition. New orleans, 17-22 November 2002.

[23] K. SubbaraJ, M.A. Dokainish. A Sutvey of Direct Time-Integation Methods in Computational Dynamics-11: Implicit Methods. Computer \& Structures, 32, 1387-1401, 1989.

[24] Y. THOMAS. Signaur et ş̧stėnes linéaires. Masson, 1992.

[25] Z.U.A WARSI. Conservation form of the Navier Stokes Equations in General Non Steady Coordinates. American Institue of Aeronautics and Astronautics Journal, 19, 240.242, 1980.

[26] K. WASHIZU. Variational Methods in Flasticity and Plasticity, Pergamon, 1982.

[27] T. WINTERGERSIE. Fluid-Structure Interaction sith STAR-CD and PERMAS using MpCCl. BENCHmark (NAFEMS Publication), April 2002.

[28] STAR-CD Methodology-User Guide. CD Adapco Group, 2003. 